\title{
Identification of novel potent human testis-specific and bromodomain-containing protein (BRDT) inhibitors using crystal structure-based virtual screening
}

\author{
NANA GAO ${ }^{1,2 *}$, JIXIA REN $^{3 *}$, LI HOU $^{2}$, YUE ZHOU $^{2}$, LING XIN ${ }^{2}$, \\ JIEDONG WANG ${ }^{2}, \mathrm{HEMING} \mathrm{YU}^{2}, \mathrm{YONG} \mathrm{XIE}^{3}$ and HUIPING WANG \\ ${ }^{1}$ Central Laboratory, Beijing Shijitan Hospital Affiliated to Capital Medical University, Beijing 100038; \\ ${ }^{2}$ Department of Reproductive Immunology and Pharamacology, National Research Institute for Family Planning, \\ Beijing 100081; ${ }^{3}$ Institute of Medicinal Plant Development, Chinese Academy of Medical Sciences \\ and Peking Union Medical College, Haidian, Beijing 100193, P.R. China
}

Received December 30, 2015; Accepted March 30, 2016

DOI: $10.3892 / \mathrm{ijmm} .2016 .2602$

\begin{abstract}
Human testis-specific and bromodomain-containing protein (hBRDT) is essential for chromatin remodeling during spermatogenesis and is therefore an attractive target for the discovery of male contraceptive drugs. In this study, pharmacophore modeling was carried out based on the crystal structure of hBRDT in complex with the inhibitor, JQ1. The established pharmacophore model was used as a 3D search query to identify potent hBRDT inhibitors from an in-house chemical database. A molecular docking analysis was carried out to filter the obtained hit compounds. A total of 125 compounds was finally selected based on the ranking order and visual examination. These compounds were further evaluated by a protein-based in vitro assay. Four compounds with new chemical scaffolds were identified to be hBRDT inhibitors. The most active of these compounds, T480, had a half maximal inhibitory concentration $\left(\mathrm{IC}_{50}\right)$ of $9.02 \mu \mathrm{M}$. The detailed analysis of the binding mode of compound T480 provides important information for the further development of novel BRDT inhibitors.
\end{abstract}

\section{Introduction}

The control of human fertility is an important global issue $(1,2)$. In contrast to the range of contraceptives available to women,

Correspondence to: Dr Huiping Wang, Department of Reproductive Immunology and Pharamacology, National Research Institute for Family Planning, 12 Da Hui Si Road, Haidian, Beijing 100081, P.R. China

E-mail: nrifp_wang@163.com

*Contributed equally

Key words: testis-specific and bromodomain-containing protein, pharmacophore modeling, molecular docking, male contraceptive contraceptive options for men are limited to condoms or a vasectomy (3). Thus, the discovery of effective, safe and reversible male contraceptives remains a challenge. Currently, the only drugs in clinical trials are testosterone analogs $(4,5)$, although there are other possible targets (e.g., GAPDHS) (6) and drugs (e.g., gamendazole) (7). To provide alternative male contraceptives, the identification of novel protein targets that may be exploited to develop novel chemical structures as male contraceptive agents is required. One such contraceptive target is the testis-specific bromodomain-containing protein (BRDT), which is a member of the bromo and extra-terminal (BET) family. The BET family of BRD proteins is composed of 4 members in mammals (BRD2, BRD3, BRD4 and BRDT), each containing 2 conserved $\mathrm{N}$-terminal bromodomains (8). BET proteins play critical roles in cellular proliferation and cell cycle progression (9). BRDT is a tissue-specific, chromatin-associated protein expressed throughout the pachytene stage in diplotene spermatocytes (10). BRDT is a key protein that participates in chromatin remodeling during spermatogenesis. The essential role of BRDT in spermatogenesis is mediated by the first bromodomain (BD1; Fig. 1A), which specifically binds the hyperacetylated histone 4 (H4Kac4) with moderate potency $(20 \mu \mathrm{M})(11)$. The loss of the BD1 of BRDT results in improper/incomplete spermatid elongation and sperm that exhibit severe morphological defects (12). The genetic analysis of BRDT has demonstrated that the selective deletion of the BD1 encoding region is sufficient to confer sterility in homozygous hypomorphic male mice (10). The lack of fulllength BRDT leads to changes in transcription and alternative splicing in spermatocytes and spermatids (12). Thus, a small molecule that specifically inhibits BRDT function represents a suitable choice for a male contraceptive. Furthermore, BRDT is not expressed in mitotically dividing spermatogonia, and thus a BRDT-specific inhibitor would not affect the spermatogonial stem cell population, theoretically making any spermatogenic impairment reversible (12).

Currently, several potent pan-BET inhibitors, such as (+)-JQ1 (13), GSK1210151A (I-BET151) (14) and PFI-1 (15) have been found. Among these, only (+)-JQ1 (Fig. 1B) has been 
reported to be an effective acetyl-lysine competitive inhibitor of BRDT. In addition, there are currently no specific BRDT inhibitors undergoing clinical trials, at least to the best of our knowledge. Therefore, pursuing and discovering more potent and selective BRDT inhibitors with new scaffolds is mandatory. High-throughput screening methods, particularly computeraided virtual screening may provide economical approaches for the rapid discovery of lead compounds with new scaffolds of specified targets from large chemical databases. Docking-based virtual screening and pharmacophore-based virtual screening are classical methods that have been widely used to identify hit compounds in drug discovery. However, previous studies have shown that the individual use of these methods generally leads to a low hit rate and low enrichment factor, as well as to a high false-positive rate $(16,17)$. On the other hand, the combined use of virtual screening methods in a hybrid protocol would overcome these drawbacks. In 2012, Matzuk et al solved the crystal structure of human BRDT (hBRDT) in complex with (+)-JQ1 (18). This structure provides a good basis for the structure-based discovery of drugs targeting hBRDT.

Thus, in this study, we have taken advantage of the crystal structure of hBRDT-JQ1 to discover novel hit compounds targeting hBRDT. Both the structure-based pharmacophore modeling and molecular docking methods were adopted for virtual screening, and the hit compounds were evaluated by a protein-based assay. The virtual screening protocol is illustrated in Fig. 2. To the very best of our knowledge, this is the first example of a successful application of virtual screening to discover novel hBRDT inhibitors.

\section{Data collection methods}

Preparation of compound database. In this study, the in-house chemical database used for virtual screening was developed by the Institute of Medicinal Biotechnology, Chinese Academy of Medical Sciences, Beijing, China. This database contains the structural information of 80,000 compounds. All the compounds were energy minimized by applying the CHARMM force field and subjected to a conformational analysis using the Polling algorithm.

Structure-based pharmacophore modeling. Pharmacophore-based methods have been widely used in virtual screening (19). Structure-based pharmacophore generation uses the spatial information of the target protein for the topological description of ligand-receptor interactions. It also provides an efficient alternative to docking-based virtual screening, while continuing to represent specific ligand-protein interactions. Moreover, it has been demonstrated that the structure-based pharmacophore approach provides more detailed information and accuracy in its description of ligand binding than ligandbased methods (20). The information about the protein structure is a good source to bring forth the structure-based pharmacophore and its use as a first screening before docking studies. As only a few hBRDT inhibitors targeting the BD1 of hBRDT have been reported (18), in this study, a structure-based pharmacophore modeling based on the crystal structure of BD1 of hBRDT in complex with the inhibitor, JQ1, was carried out using the 'Receptor-Ligand Pharmacophore Generation' protocol in Discovery Studio 3.1 (DS; Accelrys, San Diego, CA, USA) with default parameters. This protocol generates selective pharmacophore models based on receptor-ligand interactions. The crystal structure of the first bromodomain of hBRDT was retrieved from the Protein Data Bank (PDB ID: 4FLP). As the water molecule is very important in the binding site of the BET family (13), the receptor structure was prepared by retaining the water molecules and adding hydrogen atoms, as previously described (21).

According to the interactions between ligand and receptor, the features, including hydrogen acceptors (HA) and hydrophobic regions (HP), were generated through the 'Receptor-Ligand Pharmacophore Generation' protocol. In addition, the excluded volumes were involved in the pharmacophore models to improve the effectiveness of virtual screening.

Docking-based virtual screening. Since pharmacophore-based virtual screening usually suffers a higher 'false-positive' rate (22), the combined use of pharmacophore-based virtual screening with docking should lead to a reduction in the falsepositive rate. In this study, a docking analysis was carried out after the pharmacophore-based analysis to filter the virtual screening results. All of the molecular docking studies were carried out using the program genetic optimisation for ligand docking (GOLD) 4.0 (23). GOLD adopts the genetic algorithm to dock flexible ligands into the binding site of a protein. The crystal structure of BRDT complexed with JQ1 (PDB ID: 4FLP) was used as the receptor structure. The binding site was defined as a sphere containing residues within $9 \AA$ of the co-ligand JQ1, which is large enough to cover the acetyl-lysine binding pocket of the N-terminal bromodomain of BRDT (w).

Subsequently, we adjusted the docking parameters until the docked pose of JQ1 was as close as possible to the original crystallized structure in the hydrophobic acetyl-lysine binding pocket of hBRDT. The final optimized docking parameters mainly included: i) the 'number of dockings' was set to 10 without using the early termination option; ii) the 'detect cavity' was turned on; iii) the optimized positions of the polar protein hydrogen atoms were saved; iv) the GA parameter was set to 'gold default'; v) the top 10 scoring poses were saved for each compound; and vi) the default settings were used for the other parameters. The scoring function ChemPLP was used.

In vitro assay. The in vitro bioactivities of the 125 selected hit compounds were performed by a time-resolved fluorescence resonance energy transfer (TR-FRET) assay with the Cayman BRDT bromodomain 1 TR-FRET assay kit (600650; Cayman Chemical Co., Ann Arbor, MI, USA), according to the vendor's instructions. The TR-FRET assay kit is a homogeneous, TR-FRET assay method amenable to the rapid characterization of inhibitors of bromodomain/acetylated peptide interactions in a high-throughput format. The assay was performed in 384-well plates with a $20 \mu 1$ final assay volume. Briefly, the control (JQ1) and sample (positive compounds acquired from virtual screening) were incubated in $10 \mu \mathrm{l}$ of the BRDT europium chelate for $15 \mathrm{~min}$ at room temperature. Following incubation, the reaction was initiated by the addition of $5 \mu \mathrm{l}$ of the reconstituted BRDT bromodomain 1 ligand/APC acceptor mixture. The plate was then sealed with an adhesive aluminum seal and incubated at room temperature for $1 \mathrm{~h}$. The TR-FRET ratio $(670 \mathrm{~nm}$ emission/620 $\mathrm{nm}$ emission) was measured using an EnVision 2104 multilabel plate reader (PerkinElmer, 


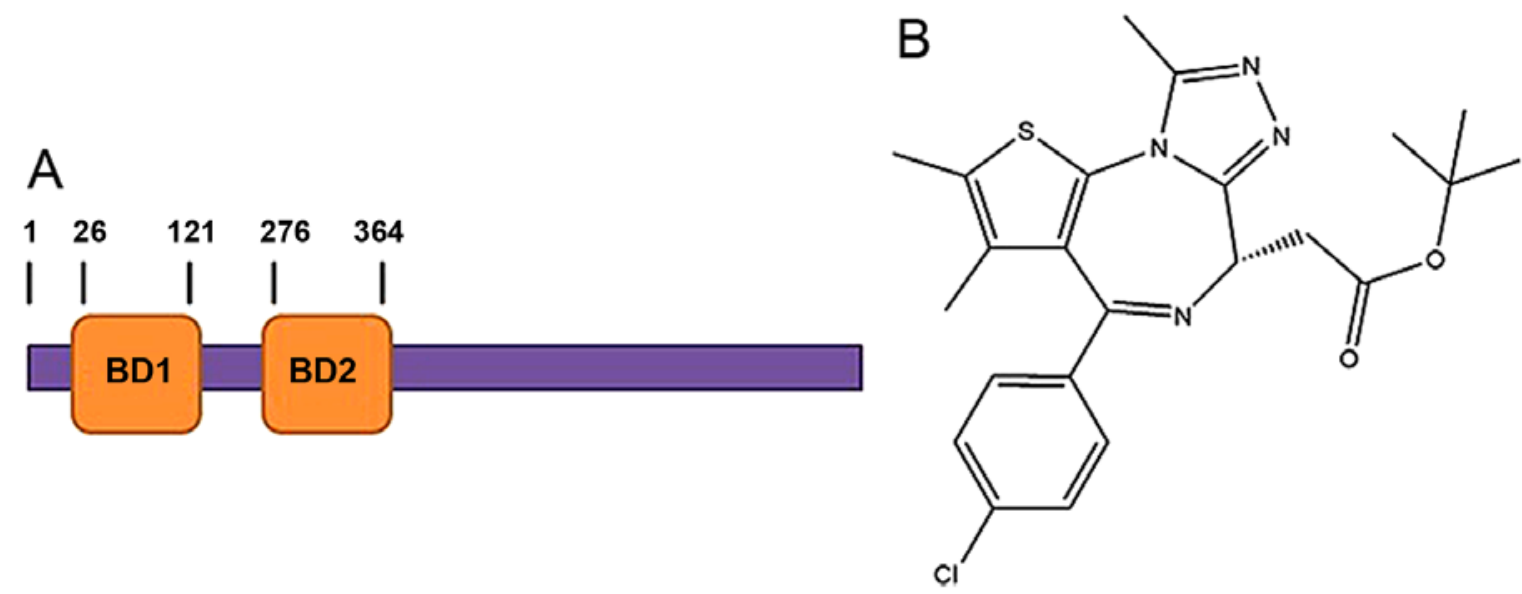

Figure 1. (A) Domain diagram of testis-specific and bromodomain-containing protein (BRDT). Sequence boundaries for recombinant BD1 are shown in bold; (B) Structure of the active (+)-JQ1 enantiomer.

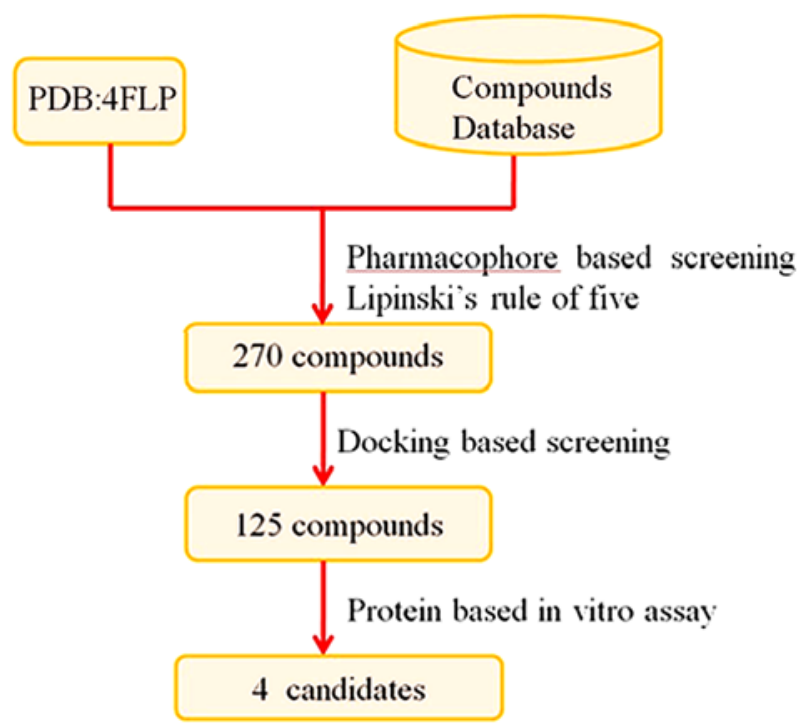

Figure 2. Flowchart of the virtual screening strategy.

Waltham, MKA, USA) in our laboratory. A plot of the TR-FRET ratio (670 $\mathrm{nm}$ emission/620 $\mathrm{nm}$ emission) vs. the inhibitor concentration on semi-log axes resulted in a sigmoidal dose-response curve typical of competitive binding assays. The data were fit to a 4-parameter logistic equation to calculate the half maximal inhibitory concentration $\left(\mathrm{IC}_{50}\right)$ values. Samples were prepared in dimethyl sulfoxide (DMSO) and the final concentration of DMSO in the assay was $<2 \%(\mathrm{v} / \mathrm{v})$.

\section{Results and Discussion}

Generation of structure-based pharmacophore modeling. The 'Receptor-Ligand Pharmacophore Generation' protocol of DS presents the chemical features which instigate key interactions between protein and ligand, as well as some excluded volume spheres corresponding to the 3D structure of protein. In this study, the 3D structures of hBRDT bound with its inhibitor, JQ1, were selected as input for structure-based pharmacophore generation. In general, the excluded volumes attempt to penalize molecules occupying steric regions that are not occupied by active molecules. The filtering of the pharmacophore with these excluded volume features provides a more selective model to reduce false-positives and a better enrichment rate in virtual screening. The parameters for the minimum features and the maximum features were set to 4 and 6 , respectively. The parameter for keeping water molecules was set to false. The 'Receptor-Ligand Pharmacophore Generation' module identified all the possible pharmacophore features based on the interactions between the acetyl-lysine binding pocket of hBRDT and JQ1. Finally, a pharmacophore model which involves 1 hydrogen bond acceptor, 4 hydrophobic features and 14 excluded volumes was obtained (Fig. 3A and B). Fig. 4A shows the pharmacophore model and residues responsible for the pharmacophore features. Fig. 4B shows the inhibitor, JQ1, mapped with the pharmacophore features. Clearly, inhibitor JQ1 is mapped very well with these features. Fig. 4C shows that the hydrogen bond acceptor corresponds to the hydrogen bond formed between the triazole ring of JQ1 and the evolutionarily conserved asparagine (ASN109). Four hydrophobic features map the different parts of JQ1; H1, H2, H3 and H4 map the methyl, thiophene ring, benzene ring and tertiary butyl, respectively. These hydrophobic features refer to the hydrophobic regions at the active site of BRDT, formed mainly by TRP50, PRO51, PHE52, LEU61, LEU63, ILE115 and MET118.

Pharmacophore-based virtual screening. The setup of the pharmacophore model was complete. Subsequently, the best pharmacophore model, including 5 chemical features and 14 excluded volumes was used as a 3D search query for retrieving potent BRDT inhibitors from an in-house chemical database $(80,000$ molecules). Only those compounds that mapped at least 3 of the 5 features were selected. These compounds were further screened using Lipinski's rule of five to make them more drug-like. A total of 270 drug-like molecules wa finally selected.

Docking-based virtual screening. To further filter the retrieved hits, the remaining 270 compounds were docked into the inhibitor binding site of BRDT using GOLD within the Discovery Studio 3.1 program package. Compounds were ranked according to the scoring function ChemPLP incorporated in 

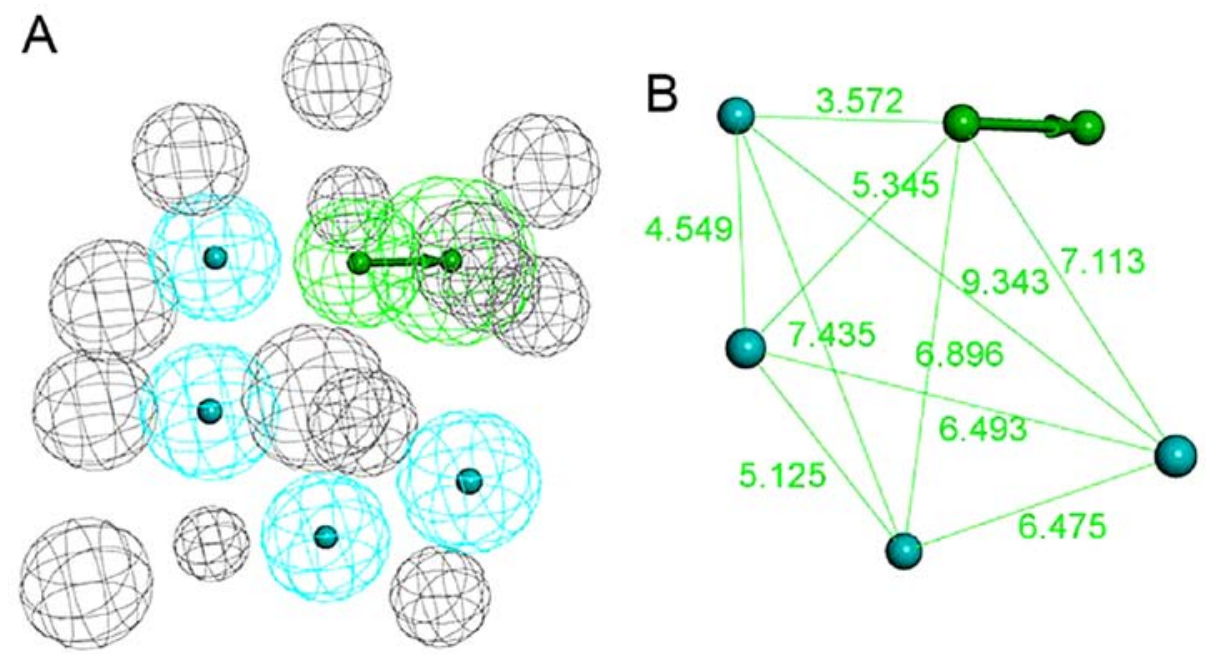

Figure 3. (A) Pharmacophore model derived from the crystal structure of testis-specific and bromodomain-containing protein (BRDT)-JQ1. The features are color coded: green, hydrogen-bond acceptor; blue, hydrophobic feature; and grey, excluded volume features. (B) 3D spatial relationship and geometric parameters of the pharmacophore model. For clarity, the excluded volume features and the location spheres of pharmacophore features are not shown.
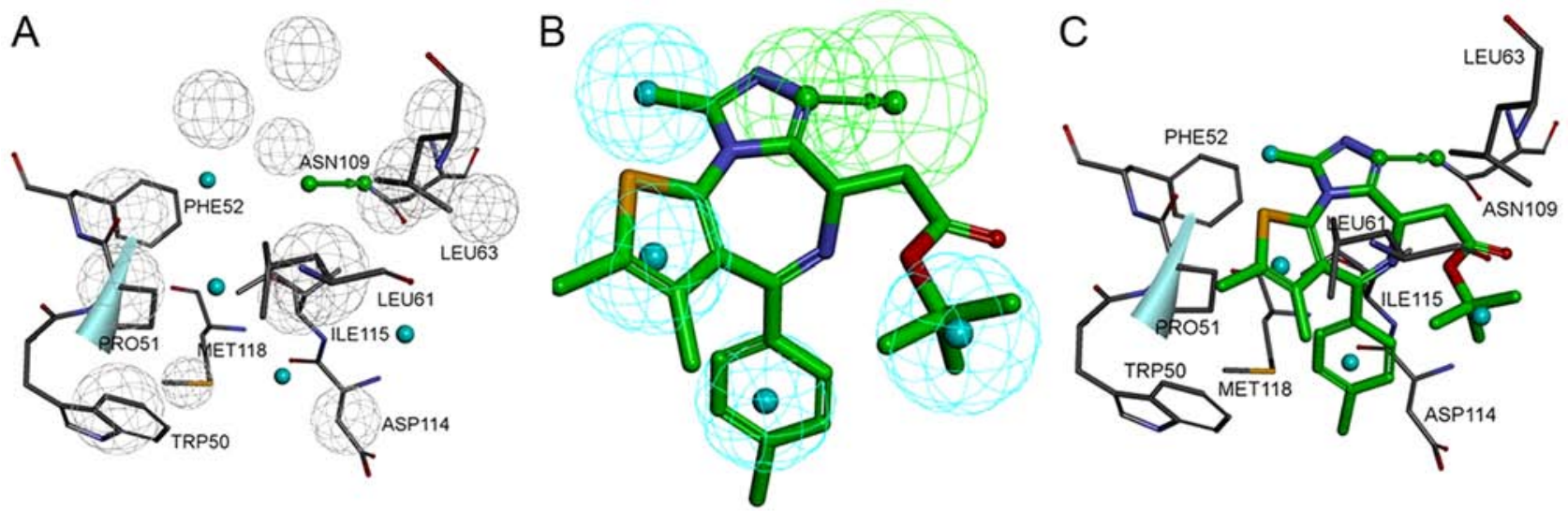

Figure 4. (A) The pharmacophore model and residues responsible for the pharmacophore features. (B) The mapping of JQ1 with the pharmacophore model. (C) The mapping of the pharmacophore model with the crystal structure of testis-specific and bromodomain-containing protein (BRDT)-JQ1. Pharmacophore features are color coded: green, hydrogen-bond acceptor; blue, hydrophobic feature; and gray, excluded volume. (B and C) For clarity, the excluded volume features are not shown.

GOLD; ChemPLP was used as the pre-evaluation of the results of various scoring functions revealed that ChemPLP was more effective than others (data not shown). Finally, 125 compounds were selected based on the ranking order and visual examination. These compounds were used in the subsequent in vitro assay.

In vitro assay. The in vitro inhibitory activities of the 125 compounds were evaluated by TR-FRET assay using JQ1 as a standard reference drug. From this assay, 4 compounds were identified to display obvious inhibitory activities with $\mathrm{IC}_{50}$ values $<33 \mu \mathrm{M}$ (Table I and Fig. 5). The fitting curves for the $\mathrm{IC}_{50}$ data of the compounds are shown in Fig. 6. These hits, which were novel small molecule inhibitors, showed structural diversity.

The detailed binding mode of the most active compound, T480, and the mapping of compound T480 with the pharmacophore model are shown in Fig. 7A and B, respectively. This compound will be analyzed in subsequent investigations,
Table I. Inhibitory rates and inhibitory activities against hBRDT.

\begin{tabular}{lcc}
\hline Compound & Inhibition rate ${ }^{\mathrm{a}}(\%)$ & $\mathrm{IC}_{50}(\mu \mathrm{M})$ \\
\hline T480 & 65.53 & $9.02 \pm 0.45$ \\
T272 & 71.23 & $12.77 \pm 0.38$ \\
T225 & 44.39 & $32.12 \pm 0.63$ \\
T323 & 74.08 & $16.85 \pm 0.59$ \\
JQ1 & $70.34^{\mathrm{b}}$ & 0.133 \\
\hline
\end{tabular}

${ }^{a}$ The inhibition rate was determined at the concentration of $100 \mu \mathrm{M}$. ${ }^{\mathrm{b}}$ The inhibition rate was determined at the concentration of $10 \mu \mathrm{M}$. hBRDT, human testis-specific and bromodomain-containing protein.

including chemical structural modification and further pharmacological studies. Fig. 7A shows that compound T480 docks tightly into the active site of hBRDT. Two hydrogen- 
<smiles>CN(Cc1cn(-c2ccccc2)nc1-c1ccc(Cl)cc1)c1nnnn1-c1ccccc1</smiles>

T480<smiles>[R22]#CCCCC(C)n1c(SCc2nnc3n(C)c(=O)c4ccccc4n23)nc2ccccc2c1=O</smiles><smiles>CN(Cc1nc2ccccc2n1C(F)F)C(=O)CCNC(=O)c1ccc([N+](=O)[O-])cc1</smiles>

T225<smiles>Cc1ccc(S(=O)(=O)N2CCCc3ccccc32)c(C)c1</smiles>

T323

Figure 5. Structures of human testis-specific and bromodomain-containing protein (hBRDT) inhibitors identified in this study.
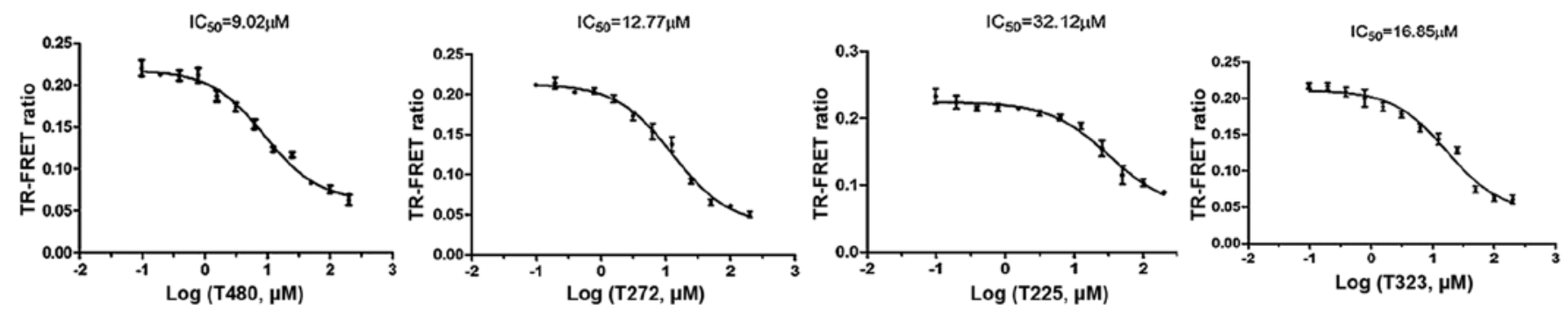

Figure 6. The fitting curves for the half maximal inhibitory concentration $\left(\mathrm{IC}_{50}\right)$ data of the compounds identified.
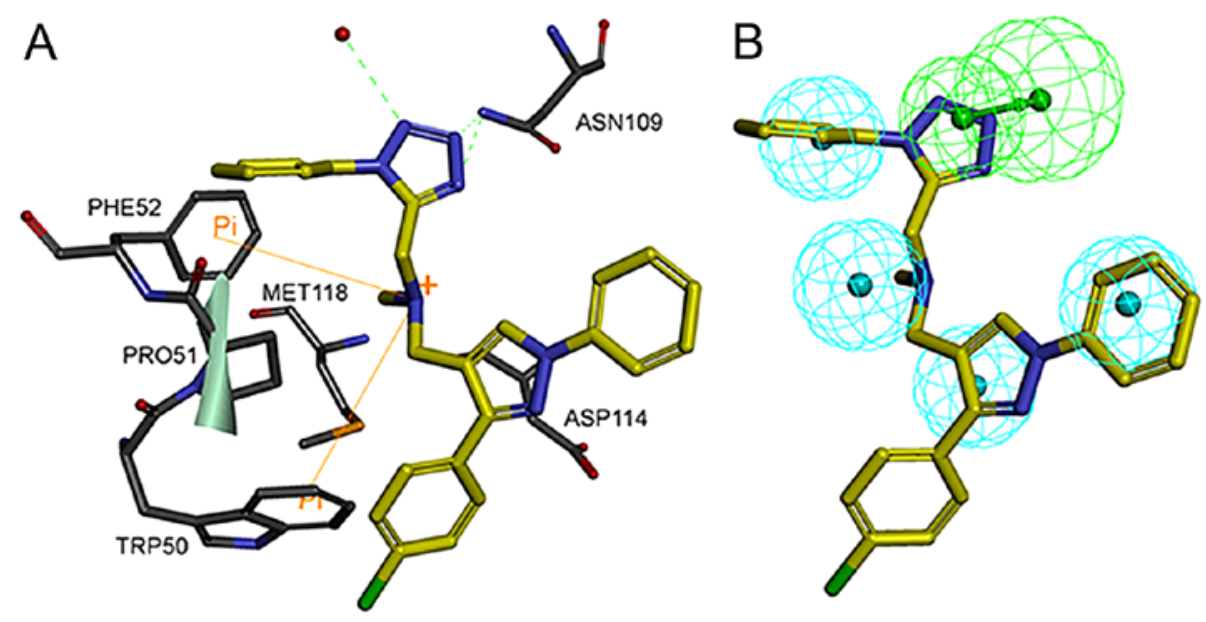

Figure 7. (A) The interaction mode between T480 and the active site of human testis-specific and bromodomain-containing protein (hBRDT) (dashed lines represent hydrogen bonds, the red dot represent the critical water molecular). (B) Mapping of the most active compound T480 with the pharmacophore model.

bond interactions are formed between compound T480 and hBRDT: one is between the tetrazole ring nitrogen atom and the amino group of the amide of the conserved ASN109 residue, whereas the other is between the other nitrogen atom on the tetrazole ring of compound T480 and the same amino group of the conserved ASN109. One hydrogen-bond interaction is formed between one nitrogen atom on the tetrazole ring of T480 and the conservative water molecule. The phenyl moieties of T480 form hydrophobic interactions with PHE52, PRO51, TRP50 and ASP114. In addition, the nitrogen of methenamine forms cation- $\pi$ interactions with PHE52 and TRP50. Fig. 7B shows that the tetrazole ring and 2 phenyl moieties of compound T480 map the hydrogen-bond acceptor feature and 2 wo hydrophobic features, respectively. The good binding pose and interactions of T480 with the active site of hBRDT provide a solid basis for their bioactivity against hBRDT.

Virtual screening resulted in multiple novel inhibitor classes targeting hBRDT. In the combined screening method, ligandprotein X-ray structure pharmacophore was used to search large databases and provided a much smaller set of compounds for docking into the active site. This method provided an efficient method of finding new leads.

In conclusion, our study demonstrates that hBRDT is a promising target in the discovery of male contraceptive drugs. In this study, both pharmacophore-based and docking-based 
virtual screening methods were developed and applied in a virtual screening of an in-house database for retrieving inhibitors of hBRDT. A total of 125 compounds were selected based on the ranking order in the virtual screening for the in vitro protein-based assays. Four novel inhibitors were identified and revealed moderate inhibitory potencies with $\mathrm{IC}_{50}$ values ranging from $9.02 \pm 0.45$ to $32.12 \pm 0.63 \mu \mathrm{M}$. The detailed analysis of the binding modes using the docking study for compound T480 showed that the inhibitor was stabilized by the formation of 2 hydrogen bonds with the key residue ASN109. Moreover, further development of these inhibitors is currently ongoing.

\section{Acknowledgements}

This study was supported by grants from the China Postdoctoral Science Foundation (no. 2014M550678 to N.G.), the Special Research Funds from the Ministry of Science and Technology of P.R. China (no. 2012GJSSJKB01 to H.W.).

\section{References}

1. Henshaw SK: Unintended pregnancy in the United States. Fam Plann Perspect 30: 24-29, 46, 1998.

2. Finer LB and Henshaw SK: Abortion incidence and services in the United States in 2000. Perspect Sex Reprod Health 35: 6-15, 2003.

3. Piccinino LJ and Mosher WD: Trends in contraceptive use in the United States: 1982-1995. Fam Plann Perspect 30: 4-10,46, 1998

4. Gu Y, Liang X, Wu W, Liu M, Song S, Cheng L, Bo L, Xiong C, Wang X, Liu X, et al: Multicenter contraceptive efficacy trial of injectable testosterone undecanoate in Chinese men. J Clin Endocrinol Metab 94: 1910-1915, 2009.

5. Hay CJ, Brady BM, Zitzmann M, Osmanagaoglu K, Pollanen P, Apter D, Wu FC, Anderson RA, Nieschlag E, Devroey P, et al: A multicenter Phase IIb study of a novel combination on intramuscular androgen (testosterone decanoate) and ora progestogen (etonogestrel) for male hormonal contraception. J Clin Endocrinol Metab 90: 2042-2049, 2005.

6. Frayne J, Taylor A, Cameron G and Hadfield AT: Structure of insoluble rat sperm glyceraldehyde-3-phosphate dehydrogenase (GAPDH) via heterotetramer formation with Escherichia coli GAPDH reveals target for contraceptive design. J Biol Chem 284: 22703-22712, 2009.

7. Aitken RJ, Baker MA, Doncel GF, Matzuk MM, Mauck CK and Harper MJ: As the world grows: contraception in the 21st century. J Clin Invest 118: 1330-1343, 2008.

8. Picaud S, Da Costa D, Thanasopoulou A, Filippakopoulos P, Fish PV, Philpott M, Fedorov O, Brennan P, Bunnage ME, Owen DR, et al: PFI-1, a highly selective protein interaction inhibitor, targeting BET Bromodomains. Cancer Res 73: 3336-3346, 2013.

9. Dey A, Nishiyama A, Karpova T, McNally J and Ozato K: Brd4 marks select genes on mitotic chromatin and directs postmitotic transcription. Mol Biol Cell 20: 4899-4909, 2009.
10. Shang E, Nickerson HD, Wen D, Wang X and Wolgemuth DJ: The first bromodomain of Brdt, a testis-specific member of the BET sub-family of double-bromodomain-containing proteins, is essential for male germ cell differentiation. Development 134: 3507-3515, 2007.

11. Morinière J, Rousseaux S, Steuerwald U, Soler-López M, Curtet S, Vitte AL, Govin J, Gaucher J, Sadoul K, Hart DJ, et al: Cooperative binding of two acetylation marks on a histone tail by a single bromodomain. Nature 461: 664-668, 2009.

12. Berkovits BD and Wolgemuth DJ: The role of the double bromodomain-containing BET genes during mammalian spermatogenesis. Curr Top Dev Biol 102: 293-326, 2013.

13. Filippakopoulos P, Qi J, Picaud S, Shen Y, Smith WB, Fedorov O, Morse EM, Keates T, Hickman TT, Felletar I, et al: Selective inhibition of BET bromodomains. Nature 468: 1067-1073, 2010.

14. Dawson MA, Prinjha RK, Dittmann A, Giotopoulos G, Bantscheff M, Chan WI, Robson SC, Chung CW, Hopf C, Savitski MM, et al: Inhibition of BET recruitment to chromatin as an effective treatment for MLL-fusion leukaemia. Nature 478: 529-533, 2011.

15. Fish PV, Filippakopoulos P, Bish G, Brennan PE, Bunnage ME, Cook AS, Federov O, Gerstenberger BS, Jones H, Knapp S, et al: Identification of a chemical probe for bromo and extra C-terminal bromodomain inhibition through optimization of a fragment-derived hit. J Med Chem 55: 9831-9837, 2012.

16. Zhang H, Xiang ML, Liang JY, Zeng T, Zhang XN, Zhang J and Yang SY: Combination of pharmacophore model, and molecular docking to identify novel inhibitors of S6K1. Mol Divers 17: 767-772, 2013.

17. Ren JX, Li LL, Zheng RL, Xie HZ, Cao ZX, Feng S, Pan YL, Chen X, Wei YQ and Yang SY: Discovery of novel Pim-1 kinase inhibitors by hierarchical multistage virtual screening approach based on SVM model,pharmacophore, and molecular docking. J Chem Inf Model 51: 1364-1375, 2011.

18. Matzuk MM, McKeown MR, Filippakopoulos P, Li Q, Ma L, Agno JE, Lemieux ME, Picaud S, Yu RN, Qi J, et al: Small-molecule inhibition of BRDT for male contraception. Cell 150: 673-684, 2012.

19. Leach AR, Gillet VJ, Lewis RA and Taylor R: Three-dimensional pharmacophore methods in drug discovery. J Med Chem 53: 539-558, 2010.

20. Steindl T and Langer T: Influenza virus neuraminidase inhibitors: generation and comparison of structure-based and common feature pharmacophore hypotheses and their application in virtual screening. J Chem Inf Comput Sci 44: 1849-1856, 2004.

21. Smellie A, Stanton R, Henne R and Teig S: Conformational analysis by intersection: CONAN. J Comput Chem 24: 10-20, 2003.

22. Yang SY: Pharmacophore modeling and applications in drug discovery: challenges and recent advances. Drug Discov Today 15: 444-450, 2010.

23. Jones G, Willett P, Glen RC, Leach AR and Taylor R: Development and validation of a genetic algorithm for flexible docking. J Mol Biol 267: 727-748, 1997.

24. Dhalluin C, Carlson JE, Zeng L, He C, Aggarwal AK and Zhou MM: Structure and ligand of a histone acetyltransferase bromodomain. Nature 399: 491-496, 1999. 